Chirurg 2020 $91: 355$

https://doi.org/10.1007/s00104-020-01147-1 Online publiziert: 21. Februar 2020

(c) Springer Medizin Verlag $\mathrm{GmbH}$, ein Teil von Springer Nature 2020

\section{Originalpublikation}

Beetz O, Söffker R, Cammann S, Oldhafer F et al (2020) Extended hepatic metastasectomy for renal cell carcinoma - new aspects in times of targeted therapy: a single-center experience over three decades. Langenbecks Arch Surg. https://doi.org/10.1007/s00423-019-018524.

Einleitung. Synchrone und metachrone Metastasierung trägt maßgeblich zur Mortalität bei Nierenzellkarzinom bei. Der Stellenwert der chirurgischen Resektion von Lebermetastasen ist dabei wenig evidenzbasiert, insbesondere seit der Verfügbarkeit gezielter medikamentöser Therapieoptionen wie dem 2006 eingeführten Rezeptor-Tyrosinkinase-Inhibitor Sunitinib.

Methodik. Bei dieser retrospektiven Analyse wurden insgesamt 40 Erwachsene betrachtet, bei denen zwischen 1993 und 2017 Leberresektionen bei histologisch bestätigten Metastasen von Nierenzellkarzinomen durchgeführt wurden. Die Operationen wurden in Resektion mit bis zu 3, bis zu 5 Lebersegmenten sowie multiviszerale Resektionen eingeteilt. Primäre Endpunkte waren krankheitsfreies Überleben („disease free survival“, DFS) und Gesamtüberleben („overall survival“, OS), sekundär wurden chirurgische Komplikationen und Dauer von Intensivpflichtigkeit und Hospitalisierung untersucht. Risiko- und protektive Faktoren für die primären Endpunkte und Unterschiede im Überleben bei Patienten vor und nach 2006 wurden untersucht.

Claus Michael · Michael Ardelt · Utz Settmacher

Klinik für Allgemein-, Viszeral- und Gefäßchirurgie, Universitätsklinikum Jena, Jena, Deutschland

\title{
Erweiterte hepatische Resektionen bei Metastasen von Nierenzellkarzinomen
}

Ergebnisse. Perioperativ wurden bei 14 Patienten extrahepatische Metastasen nachgewiesen, diese zeigten ein signifikant kürzeres medianes DFS (9,9 vs. 23,5 Monate) sowie OS (23,4 vs. 47,2 Monate) als Patienten ohne extrahepatische Tumormanifestation. Multiviszerale Resektionen waren mit signifikant mehr postoperativen Komplikationen (75,0\% vs. $32,3 \%)$ und schweren Komplikationen $(62,5 \%$ vs. $12,9 \%)$ behaftet. Die multivariable Analyse zeigte die multiviszerale Resektion als einzigen unabhängigen signifikanten Risikofaktor (HR 2,966, 95\%-CI 1,035-8,495) für schlechteres DFS, für das OS wurden auch multiviszerale Resektionen als unabhängiger Risikofaktor (HR 9,851, 95\%CI 2,715-35,737) und ein längerer Abstand zwischen Nephrektomie und Metastasenresektion (HR 0,971, 95\%CI 0,956-0,987) als unabhängiger protektiver Faktor identifiziert. Patienten, die nach 2006 operiert wurden $(n=15)$, hatten ein signifikant besseres medianes Gesamtüberleben (45,2 vs. 27,5 Monate), obwohl sie durchschnittlich deutlich älter waren (66,9 vs. 57,2 Lebensjahre).

\section{Kommentar}

Die hier vorgestellte Studie untersucht protektive Faktoren und Risikofaktoren bei Patienten mit Leberresektionen bei hepatisch metastasierenden Nierenzellkarzinomen und vergleicht das krankheitsfreie Überleben und Gesamtüberleben vor und nach Einführung des bei Nierenzellkarzinomen effektiven Rezeptor-Tyrosinkinase-Inhibitors Sunitinib. Einschränkungen in der Aussagekraft ergeben sich aus der kleinen
Fallzahl von 40 Patienten und dem retrospektiven Charakter der Studie ohne Kontrollgruppe. Das maximale Followup der nach 2006 operierten Patienten ist kürzer, da zum Zeitpunkt der Analyse noch 10 dieser Patienten am Leben waren. Die Autoren der Studie unterstreichen den Stellenwert der Resektion bei hepatisch metastasierten Nierenzellkarzinomen und betonen die Notwendigkeit der individuellen Therapieentscheidung bei Patienten mit der Möglichkeit multiviszeraler Resektionen wegen erheblicher Komplikationen und Mortalität. Während die Arbeit keinen statistisch belastbaren Nachweis über die Verbesserung der Prognose von Patienten nach Resektion hepatischer Metastasen durch die Einführung der gezielten Therapie mit Rezeptor-Tyrosinkinase-Inhibitoren liefern kann, zeigt sich doch ein Trend, der mit längerem Follow-up in dieser Gruppe deutlicher werden könnte.

\section{Korrespondenzadresse}

PD. Dr. Dr. Michael Ardelt

Klinik für Allgemein-, Viszeral- und Gefäßchirurgie, Universitätsklinikum Jena Am Klinikum 1, 07747 Jena, Deutschland Michael.Ardelt@med.uni-jena.de

Interessenkonflikt. C. Michael, M. Ardelt und U. Settmachergeben an, dass kein Interessenkonflikt besteht. 